\title{
Ligneous conjunctivitis in a patient with Crohn's disease
}

\author{
Graciana Fuentes-Páez' \\ José $M$ Herreras' \\ $M$ del C Méndez ${ }^{2}$ \\ MA Saorni ${ }^{1,2}$ \\ 'Ocular Immunology and Uveitis \\ Unit, Institute of Ophthalmobiology \\ (IOBA), University of Valladolid, \\ Valladolid, Spain and Hospital \\ Universitario de Valladolid, Spain; \\ ${ }^{2}$ Ocular Pathology and Lab Registry \\ "Miguel N. Burnier", Institute of \\ Ophthalmobiology (IOBA), University \\ of Valladolid, Valladolid, Spain
}

Objective: Report the case of a patient with Crohn's disease and ligneous conjunctivitis. Methods: Interventional case report of a 27-year old female patient, with a 4-year history of Crohn's disease, was seen for right eye conjunctival ulcer after resolved chemical trauma. Conjunctival biopsy was performed for histopathology, immunofluorescence microscopy, and immunohistochemistry testing.

Results: Microbiology tests were negative for bacteria and fungi. PCR was negative for atypical mycobacteria and Herpes. Ocular Crohn's disease, Whipple disease, and amyloidosis were ruled out. Immunofluorescence microscopy revealed characteristic $\operatorname{IgG} \kappa$ and $\lambda$ light chain deposits, and fibrin deposition was confirmed through Lendrum's Martius, Scarlet, and Blue technique. Endogenous plasminogen levels were normal. Recurrent ulcers did not resolve after treatment with infliximab, but only after four surgeries, topical steroids, $1 \%$ cyclosporine, heparin (5000 units $/ \mathrm{ml})$, and hyaluronidase $(1.5 \mathrm{mg} / \mathrm{ml})$. After 9 months of follow-up, nasal symblepharon was observed as sequela.

Conclusions: We report the case of a patient with unilateral ligneous conjunctivitis, triggered by chemical injury, and Crohn's disease.

Keywords: Crohn's disease, immunofluorescence microscopy, ligneous conjunctivitis, plasminogen, ulcerative conjunctivitis

\section{Introduction}

Ligneous conjunctivitis (LC) is a rare form of chronic conjunctivitis characterized by the development of fibrin-rich, woody-like pseudomembraneous tissue in the tarsal conjunctiva (Schuster and Seregard 2003). It occurs in association with external irritants and ocular surgery (Schuster and Seregard 2003).

Plasminogen deficiency has recently been found to be the most common cause of LC, and the lesions are the result of an impaired wound healing response (Schuster and Seregard 2003). LC seems to be the most common symptom of this systemic disorder, since it usually occurs with other mucosal involvement (Schuster and Seregard 2003).

Crohn's disease (CD) is an inflammatory bowel disease with ocular involvement as an extraintestinal manifestation. The eye disease commonly presents as anterior uveitis and, rarely, with naevus and conjunctival or lid ulcers (Lendrum et al 1962; Schuettenberg 1991; Hegab and al-Mutawa 1994; Zoli et al 1997; Diaz-Valle et al 2004). Based on clinical evolution and histopathology findings, we report a rare case of a patient with CD and LC triggered by chemical injury.

\section{Case report}

A 27-year-old female patient presented at our center with a 6-week history of right eye pain and ulceration. The symptoms appeared one month after a resolved conjunctival chemical burn with $17 \%$ hydrochloric acid. The recurrent corneal and conjunctival 
ulcers progressed despite topical corticosteroids, 1\%, cyclosporine A (CsA), antibiotics, and artificial tears. The patient also had a 4-year history of aggressive CD, confirmed by intestinal biopsy, which required immunosuppressive therapy with azathioprine, anti-TNF-alpha treatment, and intestinal resection. Her CD had been stable for last 6 months.

Visual acuity was 20/200 for her right eye and 20/20 for the left eye. Our initial slit-lamp examination of her right eye revealed plaque-like ulcerations in the nasal bulbar conjunctiva and in the inferior and superior tarsal conjunctivas (Figure 1A, B). The left eye was normal. Systemic workup ruled out sarcoidosis, systemic vasculitis, rickettsias, brucella, syphilis, chlamydia, Lyme disease, and HIV.

Bulbar, upper, and lower fornix conjunctival resections were performed on the affected eye. Tissue specimens from the resection were processed for special stains including Gram and acridine orange for bacteria, calcofluor and periodic acid Schiff (PAS) stains for fungi and/or Whipple disease, and Congo red for amyloidosis. The presence of atypical mycobacteria and Herpes were determined by polymerase chain reaction (BIOTOOLS B and M Labs, Madrid, Spain). All of the tests were negative.

Tissue sections for routine histopathological examination were stained with hematoxylin and eosin (H\&E) and PAS stains. Fibrin deposits were assessed by Lendrum's Martius, Scarlet, and Bluetechnique (MSB) technique (Lendrum et al 1962).

Immunofluorescence microscopy for IgG and $\kappa, \lambda$ light chain deposits was performed with polyclonal antihuman antibodies from BioGenex Laboratories (San Ramon,
CA, USA) and DAKO Corp. (Carpintería, CA, USA), respectively. The secondary antibody was FITC-conjugated anti-rabbit IgG (Sigma, St. Louis, MO, USA).

Histopathology reported surface ulcerations with an underlying thick band of hyaline-like eosinophilic material, granulation tissue, and lymphocytic infiltration (Figure 2A). Abundant fibrin deposition was confirmed with the MSB technique (Figure 2B). No granulomas were found. Histopathology and IF findings were compatible with LC. Her systemic plasminogen values were tested and "verbally reported" within normal limits $(0.7-1.0 \mathrm{U} / \mathrm{ml})$, on two separate occasions.

After the conjunctival resection, her symptoms and signs resolved, but then recurred two weeks later (Figure 1C). Her gastroenterologist indicated treatment with 3 doses of inflix$\mathrm{imab}$ (5 mg/kg/day, Remicade, Schering-Plough, Kenilworth, NJ), but ocular signs did not improve. A second surgical excision was performed and treatment with $1 \%$ CsA three times a day (tid) initiated.

Three weeks later, a third excision was performed, since the lesion recurred, became very painful, and progressed toward the inferior palpebral skin. Otorrhinolaryngology evaluation was solicited and reported as normal.

Topical heparin (5000 units/ml) tid and fluorometholone tid was added. Due to further progression, a fourth excision was performed one month later, and topical hyaluronidase $(1.5 \mathrm{mg} / \mathrm{ml})$ tid added. Six months after treatment, the lesions resolved but an inferior nasal symblepharon developed as sequela, after a 9-month follow-up. (Figure 1D).

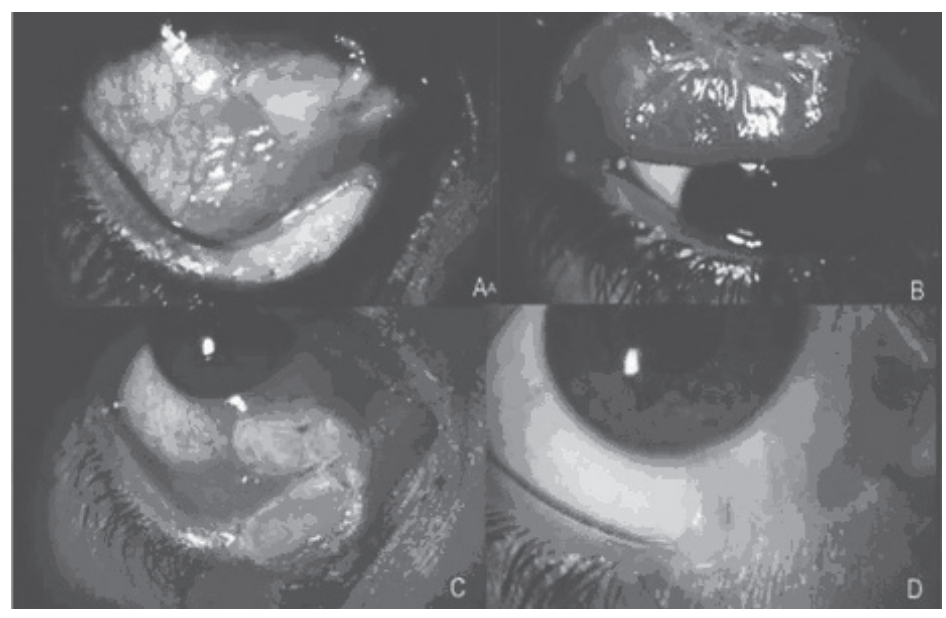

Figure I Initial right eye slit-lamp examination. The nasal bulbar and inferior conjunctivas (A) and the superior tarsal conjunctiva (B) had plaque-like ulcerations. The initial conjunctival ulceration was followed by a recurrence of the inferior tarsal ulcer (C).A nasal symblepharon remained as a sequela after a 9-month followup (D). 


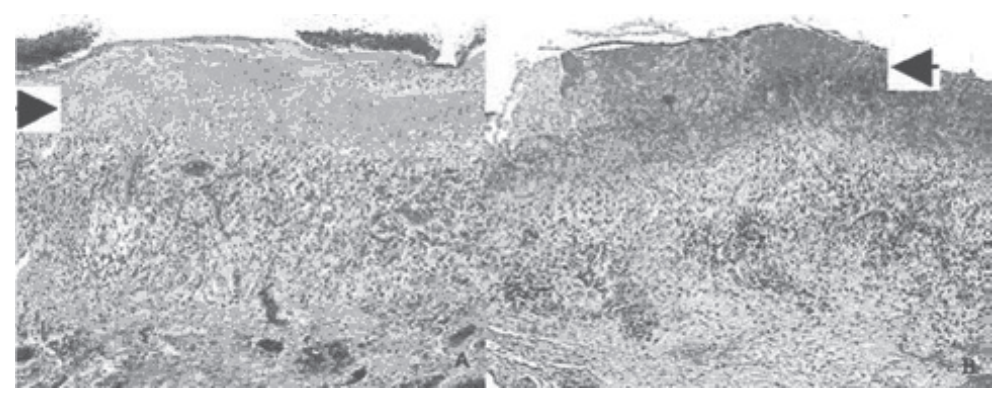

Figure 2 Histopathology of conjunctival biopsies. The superficial ulceration had an underlying thick band of hyaline-like eosinophilic material, probably composed of fibrin (arrow). Granulation tissue and lymphocytic infiltration were apparent in H\&E (A) and fibrin (arrow) was confirmed with Lendrum staining (B). The tissue sample was negative for Congo red.

\section{Discussion}

Ocular involvement, as an extraintestinal manifestations of inflammatory bowel disease, occurs in less than $12 \%$ of cases (Ghanchi and Rembacken 2003). It manifests as uveitis, episcleritis, scleritis, nonspecific follicular conjunctivitis, lid marginal ulcers, during active or inactive disease (Salmon et al 1991; Schuettenberg 1991; Hegab and al-Mutawa 1994; Zoli et al 1997; Ghanchi and Rembacken 2003; Diaz-Valle et al 2004). She had been asymptomatic for over one year with no treatment and remained as such throughout her ocular disease.

Previously reported cases of lid margin and conjunctival ulcers in CD have resolved after systemic and topical corticosteroid treatment (Hegab and al-Mutawa 1994; Diaz-Valle et al 2004). We initially believed these conjunctival ulcers were due to her $\mathrm{CD}$, which is why infliximab therapy was administered, after consulting with her gastroenterologist, however she did not respond. In addition, histopathology findings were more compatible with LC, since no granulomas were reported, which could have been triggered by previous chemical injury (Schuster and Seregard 2003).

$\mathrm{LC}$ is a rare disease, with a prevalence of 1.6 per million in Europe, and due to the inheritance of an autosomal recessive gene (Schuster and Seregard 2003). It involves a great variety of systemic manifestations including periodontitis, renal calculi, vulvovaginitis, respiratory disease, otitis media, dermatologic lesions, and hydrocephalus, among others (Schuster and Seregard 2003). Our patient did not report any of these systemic manifestations other than an ocular chemical burn one month before the lesions appeared.

LC patients may have decreased levels of plasminogen, which arrests wound healing at the granulation tissue formation stage, and subsequently forms a fibrin-rich pseudomembranous tissue (Schuster and Seregard 2003). However, normal serum plasminogen levels do not exclude
LC because it is not a diagnostic criterion, but a predisposing factor, as would be in this case.

Other triggers for the development of LC include exposure to chemical irritants and conjunctival surgery (Schuster and Seregard 2003). In this patient, exposure to chemical irritants could have triggered the LC, which recurred after multiple surgical interventions.

This patient's conjunctival ulcers healed after topical CsA, heparin, and hyaluronidase therapy, which have been reported as effective treatments for LC (Schuster and Seregard 2003).

The fact that conjunctival cicatrizing sequela is not characteristic of LC also challenged our initial diagnosis, although corneal scarring has been reported as a LC sequela, however the previous chemical burn strongly supported this diagnosis (Hidayat and Riddle 1987).

Histopathologically, LC is characterized by subepithelial, eosinophilic, amorphous material admixed with acute and chronic inflammatory cells, positive PAS staining, and negative Congo red staining (Hidayat and Riddle 1987; Holland et al 1989; Klebe et al 1999). In this case histopathology findings, abundant fibrin deposition, chronic inflammation with granulation tissue and lymphocytes, absence of granulomas, and negative Congo red staining, coincided with LC (Hidayat and Riddle 1987; Holland et al 1989; Klebe et al 1999; Schuster and Seregard 2003).

Given the history of her immunobiological disease, triggering chemical burn, clinical progression despite infliximab therapy, characteristic histopathology, multiple recurrences, and resolution after combined surgical and topical treatments, $\mathrm{LC}$ was our final diagnosis. The $\mathrm{LC}$ in this $\mathrm{CD}$ patient was refractory to conventional treatments, however the coexistence between both diseases can be considered as casual.

We present a rare case of LC, triggered by conjunctival chemical injury, casually coexisting in a patient with $\mathrm{CD}$. 


\section{Acknowledgments}

Dr. S Seregard of St. Erik's Eye Hospital, Stockholm Sweden, for performing the MSB technique for fibrin deposition confirmation. Graciana Fuentes-Páez was supported by a grant from Carolina Foundation, Ministry of Foreign Affairs, Spain. The authors have no conflict or commercial interest.

\section{References}

Diaz-Valle D, Benitez del Castillo JM, Fernández Acenero MJ, et al. 2004. Bilateral lid marginal ulcers as the inicial manifestation of Crohn's disease. Am J Ophthalmol, 138:292-4.

Ghanchi FD, Rembacken BJ. 2003. Inflammatory bowel disease and the eye. Surv Ophthalmol, 48:663-76.

Hegab SM, al-Mutawa SA. 1994. Conjunctival ulcer in a patient with Crohn's disease. Ophthalmic Surg, 9:638-9.

Hidayat AA, Riddle PJ. 1987. Ligneous conjunctivitis. A clinicopathologic study of 17 cases. Ophthalmology, 94:949-59.
Holland EJ, Chan CC, Kuwabara T, et al. 1989. Immunohistologic findings and results of treatment with cyclosporine in ligneous conjunctivitis. Am J Ophthalmol, 107:160-6.

Klebe S, Walkow T, Hartmann C, et al. 1999. Immunohistological findings in a patient with unusual late onset manifestation of ligneous conjunctivitis. Br J Ophthalmol, 83:878-9.

Lendrum AC, Slidders W, Fraser DS, et al. 1962. Studies on the character and staining of fibrin. J Clin Pathol, 15:401-13.

Salmon JF, Wright JP, Murray AD. 1991. Ocular inflammation in Crohn's disease. Ophthalmology, 98:480-4.

Schuettenberg SP. 1991. Nodular scleritis, episcleritis, and anterior uveitis as ocular complications of Crohn's disease. J Am Optom Assoc, $62: 377-81$.

Schuster V, Seregard S. 2003. Ligneous conjunctivitis. Surv Ophthalmol, 48:369-88.

Zoli G, Care M, Parazza M, et al. 1997. Ocular naevus and hyperaemia with dilation of the conjunctival vessels: an early manifestation of Crohn's disease? Ital J Gastroenterol Hepatol, 29:270-2. 